\title{
LETTER TO THE EDITOR CRISPR-Cas12a has both cis- and trans-cleavage activities on single-stranded DNA
}

Cell Research (2018) 28:1-3; https://doi.org/10.1038/s41422-0180022-x

\section{Dear Editor,}

The CRISPR-associated protein Cas12a (previously known as Cpf1), which is an endonuclease from the type V-A CRISPR system, has been applied in both in vivo genome editing and in vitro DNA assembly. ${ }^{1-3}$ Cas $12 a$ is guided by a single CRISPR RNA (crRNA) with a T-rich protospacer adjacent motif (PAM) sequence to cleave double-stranded DNA (dsDNA) targets, generating sticky ends. Different from Cas9, Cas12a cleaves both the target and non-target strands of a targeted dsDNA by a single active site in the RuvC catalytic pocket ${ }^{4-6}$ (Supplementary information, Figure S12a). Besides, Cas12a also processes precursor crRNAs to generate mature crRNAs. ${ }^{7}$ However, the cleavage activity of Cas12a on single-stranded DNA (ssDNA) targets is less understood.

To investigate the ssDNA cleavage feature of Cas12a, we employed FnCas12a to cleave short ssDNAs that were labelled with 5(6)-carboxyfluorescein (FAM) on the $3^{\prime}$ terminus and found that the ssDNA cleavage sites were near the 22 nd base (i.e., from the 21 st to the $23 \mathrm{rd}$ ), counting from the first $3^{\prime}$-base that was paired with the crRNA guide sequence (Supplementary information, Figure S1a and b and Tables S2 and 3). The cleavage did not require the existence of a PAM sequence in the targeted ssDNA (Supplementary information, Figure S1a and b). In addition, the same cleavage sites were obtained with crRNAs having guide sequences as short as 10 nucleotides (nt) (Supplementary information, Figure S1c and d), which indicates that Cas12a could cleave ssDNA at sites outside of the recognition sequence. We then tested Cas12a cleavage efficiency on ssDNA and dsDNA substrates, and cleavage of ssDNA was slower than that of dsDNA (Supplementary information, Figure S1e and f), whose PAM sequence may account for the higher efficiency.

We also performed the Cas12a cleavage experiment with a ssDNA target labelled at its $5^{\prime}$ terminus (target-DNMT1-3-R-FAM$\left.5^{\prime}\right)$. Surprisingly, no cleaved bands were observed at the predicted size $(20 \mathrm{nt})$, but short $(<6 \mathrm{nt})$ FAM-labelled products were generated (Fig. 1a). After careful analyses of experimental conditions, we found that only the ternary complex of Cas12a/ crRNA/targeted ssDNA (or targeted dsDNA) was able to cleave the 5'-labelled target ssDNA (target-DNMT1-3), generating short FAM-labelled products (Fig. $1 \mathrm{~b}$ and Supplementary information, Figure S2). The ternary complex also promiscuously cleaved collateral ssDNAs that had no complementarity to the crRNA guide sequence in the reaction system, generating short products (Fig. 1c and Supplementary information, Figure S3). As it is difficult to distinguish the precise length of the short transcleavage products via polyacrylamide gel electrophoresis, the FAM-labelled short products were purified and analysed by liquid chromatography-mass spectrometry. The results showed that 5'-FAM-labelled substrates were mainly trans-cleaved to $4 \mathrm{nt}$, while 2-nt products were observed for $3^{\prime}$-FAM-labelled substrates (Supplementary information, Figure S4).
We called the promiscuous cleavage of collateral ssDNAs transcleavage to distinguish it from the programmable on-target cleavage of target ssDNA (namely, cis-cleavage), and the proposed ssDNA cleavage processes were illustrated in Fig. 1d. When the ssDNA substrate was labelled at the $5^{\prime}$ terminus, the cis-cleaved $5^{\prime}$-labelled ssDNA products became collateral ssDNAs in the reaction system and were subsequently trans-cleaved into short products, explaining the observed cleavage pattern for $5^{\prime}$-labelled ssDNA substrate. We observed the trans-cleavage products in addition to the cis-products for short 3'-labelled targeted ssDNAs (Fig. 1a). The majority of the ternary complex most likely remained bound to the targeted ssDNAs after cis-cleavage, protecting the labelled $3^{\prime}$-terminus from exposing the trans-activity sites of the Cas12a ternary complex.

Next, we tested nine randomly selected Cas $12 \mathrm{a}$ proteins from different species in addition to the above tested FnCas12a (Supplementary information, Figures S5, 6a and Tables S1, 4 and 5), and all Cas12a proteins exhibited endonuclease activity on plasmid dsDNA (Supplementary information, Figure S6b), cis- (Supplementary information, Figure S6c) and trans-cleavage activities on ssDNA (Supplementary information, Figure S6d). This indicates that the cis- and trans-cleavage activities on ssDNA might be ubiquitous among Cas12a proteins.

When shortened targeted ssDNAs were tested, complexes with 18-nt target ssDNAs that lacked a cleavage site also showed transcleavage activity (Supplementary information, Figure S7a), indicating that cis-cleavage was not a prerequisite for trans-cleavage activity. Trans-cleavage was implemented by the endonuclease activity of the complex, as circular ssDNA (M13mp18) could also be trans-cleaved (Supplementary information, Figure S7b). Moreover, we found that all tested Cas12a complexes except the AsCas12a complex had trans-cleavage activity on collateral dsDNAs (Figure S8), and the activity of the LbCas12a, BoCas12a and Lb4Cas12a complexes was much higher.

To identify key residues involved in SsDNA cleavage of both targeted and collateral ssDNAs, we mutated several candidate residues in FnCas12a to alanines, including those related to the RNase activity (H843, K852 and K869) ${ }^{7}$ and those responsible for dsDNA cleavage (D917, E1006, D1255 and R1218) (refs. ${ }^{1,7-9}$ and Supplementary information, Figure S9). Both cis- and transcleavage of ssDNA were unaffected in the RNase activity-related mutants (Supplementary information, Figure S10), but the activities were completely lost or remarkably decreased with mutations in either the RuvC domain (D917A, E1006A and D1255A mutations in FnCas12a) or the Nuc domain (R1218A mutation in FnCas12a) (Fig. 1e, f). Recent studies showed that the RuvC catalytic pocket of both $\mathrm{C} 2 \mathrm{C} 1$ and Cas12a was responsible for the cleavage of both strands of targeted dsDNA, ${ }^{4-6}$ leading us to propose that targeted ssDNAs were cis-cleaved by this catalytic pocket (Supplementary information, Figures S11a, b, d, e and $\mathrm{S} 12 \mathrm{c}$ ). Moreover, according to the structure of the C2c1-crRNAexcess DNA complex (Supplementary information, Figure S11c), ${ }^{4}$ trans-cleavage of collateral ssDNAs could also be achieved by the same catalytic pocket (Fig. 1g, and Supplementary information, 
a

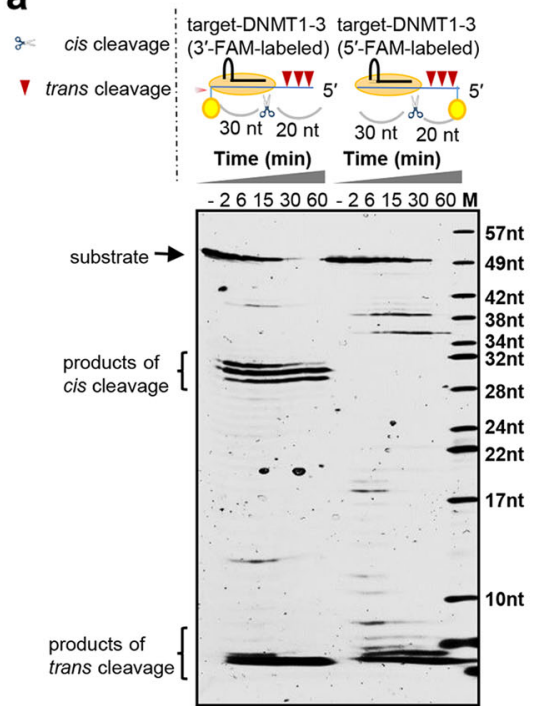

d

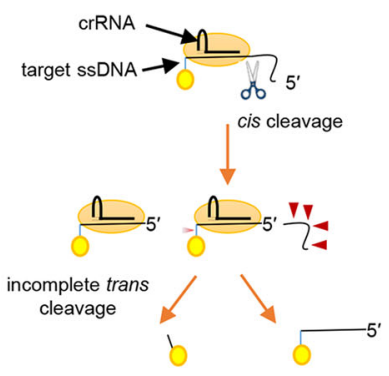

e Cas12a/crRNA-T1/target-T1(ssDNA)
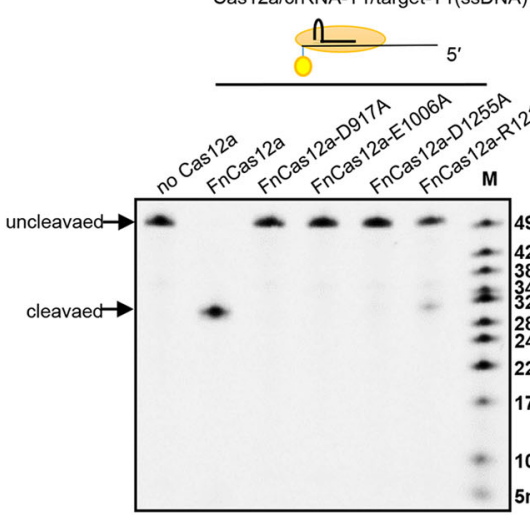

b

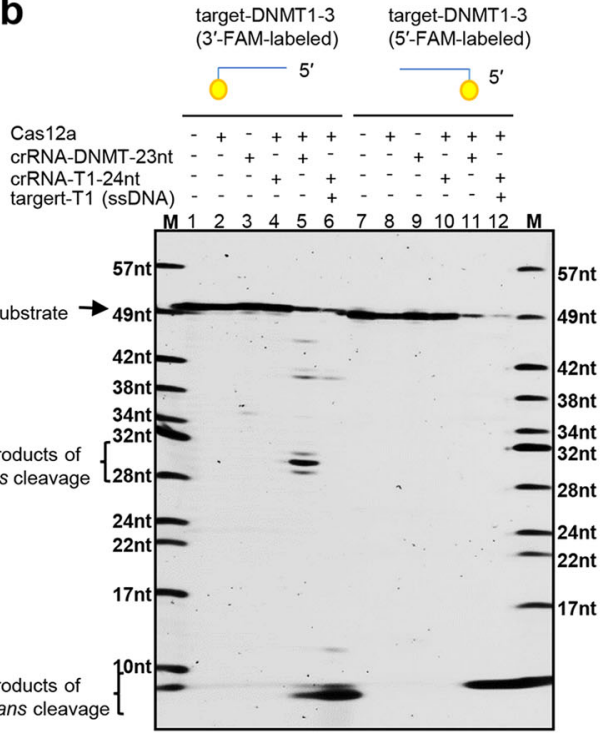

C

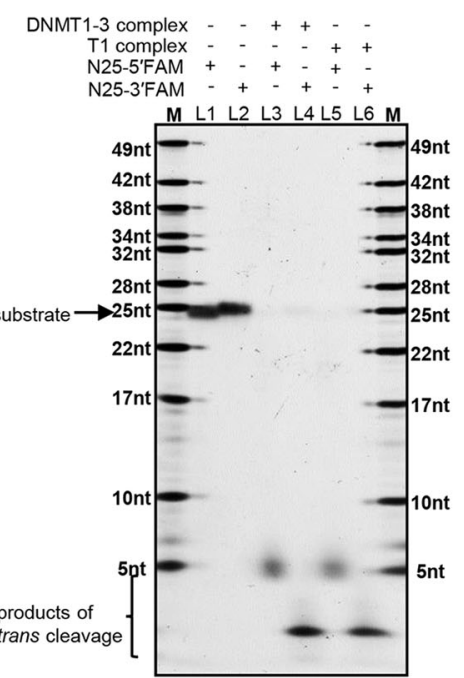

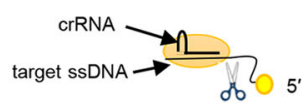

cis cleavage

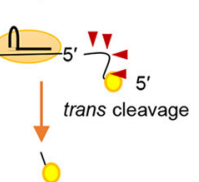

f
target-DNMT1-3 Cas12a/crRNA-T1 (collateral ssDNA) /target-T1(ssDNA) $5^{\prime} \stackrel{1}{1}$

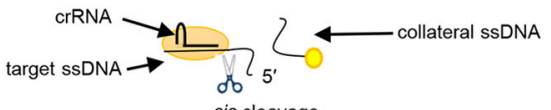

cis cleavage

$\underbrace{}_{\text {trans cleavage }}$

g

\section{Cas12a}

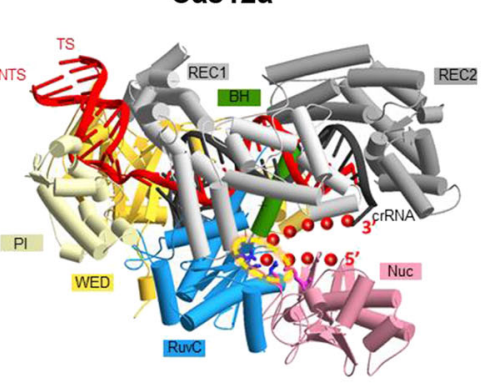

Fig. 1 Determination of the ssDNA cleavage activities of the complex of Cas12a/crRNA/target DNA. a Time-course experiment of ssDNA (target-DNMT1-3) cleavage with crRNA-DNMT-23nt. The 3'-end FAM-labelled ssDNA was cleaved at expected sites (left); however, cleavage of 5'-FAM-labelled ssDNA generated no expected products $(20 \mathrm{nt})$ but short oligonucleotides $(<6 \mathrm{nt})$. b With a target-specific crRNA of crRNA-DNMT-23nt, Cas12a cleaved the 3'-FAM-labelled target-DNMT1-3 (ssDNA) at expected sites but cleaved the 5'-FAM-labelled targetDNMT1-3 (ssDNA) to short oligonucleotides. Collateral single-stranded target-DNMT1-3 (either 5'- or 3'-FAM-labelled) was cleaved to short oligonucleotides upon the formation of the ternary complex of Cas12a/crRNA-T1-24nt/target-T1 DNA. (c) Cleavage of random collateral ssDNA by the ternary complex of Cas $12 \mathrm{a} / \mathrm{crRNA} /$ target DNA. Random short single-stranded oligonucleotides ( $25 \mathrm{nt}$ ) were labelled with FAM at either the $5^{\prime}$-end or the $3^{\prime}$-end, obtaining N25-5'FAM and N25-3'FAM, respectively. The labelled random oligonucleotides were cleaved by the complex of Cas12a/crRNA-DNMT-23nt/target-DNMT1-3 (ssDNA) in lanes 3 and 4 or by the complex of Cas12a/crRNA-T1-24nt/target-T1 (ssDNA) in lanes 5 and 6 , respectively. Lanes 1 and 2 showed synthesized random oligonucleotides that were labelled at the $5^{\prime}$-end and $3^{\prime}$-end, respectively. $\mathbf{d}$ Illustration of both cis- and trans-cleavage by the Cas12a complex in a-c. e Cis-cleavage of ssDNA (target-T1-R-FAM) by FnCas12a and its mutants. Mutations in D917, E1006, D1255 and R1218, four residues that are associated with the DNase activity, were generated. All tested mutants showed either completely lost or largely decreased cis-cleavage activity on target ssDNA. $\mathbf{f}$ Trans-cleavage of the 3'-FAM-labelled collateral ssDNA (target-DNMT1-3-R) by the ternary complexes of FnCas12a or its mutants (D917A, E1006A, D1255A and $\mathrm{R} 1218 \mathrm{~A}$ in FnCas12a). The trans-cleavage activity on collateral ssDNA was completely lost in E1006A and decreased in other mutants. $\mathbf{g}$ The ternary complex of Cas12a (PDB: 5B43) with proposed collateral ssDNA. Red dots represented the proposed positions of collateral ssDNA. Molecular graphic images were prepared using CueMol (http://www.cuemol.org). DNA was coloured in red, RNA was coloured in black and the RuvC catalytic pocket was indicated by dashed yellow circles 
Figure $\mathrm{S} 12 \mathrm{~b}$ and $\mathrm{d}$ ) in the Cas12a ternary complex. The RuvC catalytic pocket is consistently exposed in the bilobed structure of the ternary Cas12a complexes ${ }^{8}$ (Supplementary information, Figure S11f) but not in the structurally dynamic Cas12a monomer ${ }^{9}$ nor in the triangle-shaped binary structure ${ }^{9}$ (Supplementary information, Figure S11g), allowing for trans-cleavage of collateral ssDNAs.

Taken together, we here show that the cleavage activities on ssDNAs, including both cis- and trans-cleavage, are ubiquitous among Cas12a proteins, and proposed cleavage models are shown in Supplementary information, Figure S12. Notably, Cas12a is so far the first characterized Cas protein whose ternary complex has trans-ssDNA cleavage activity. Considering the fact that a large number of single-stranded viruses exist in the environment, Cas12a may get the ssDNA cleavage activity during evolution and may function as a powerful tool to prevent the invasion of foreign ssDNAs. In addition to Cas12a, Cas13a (previously known as C2c2), an RNA-guided and RNA-targeting CRISPR effector from the class 2 type VI CRISPR system, was found with the trans-cleavage activity on RNA. ${ }^{10-12}$ Recently, this characteristic of Cas13a was successfully employed for rapid and sensitive nucleic acid detection. ${ }^{13}$ Therefore, the trans-cleavage activity of Cas12a characterized in this study may also be utilized in potential biotechnological applications in a similar manner. Since different Cas12a complexes show various trans-cleavage activity on dsDNA, the in-depth mechanism needs further investigation.

\section{ACKNOWLEDGEMENTS}

We thank Chao Lei and Qi Li for their helpful discussion. This work was supported by grants from the Strategic Priority Research Program of the Chinese Academy of Sciences (XDB19040200), the Youth Innovation Promotion Association CAS (2017322) and the National Natural Science Foundation of China (31421061, 31430004 and 31300031).

\section{ADDITIONAL INFORMATION}

Supplementary information accompanies for this paper at https://doi.org/10.1038/ s41422-018-0022-x.

Competing interests: The authors declare no competing interests.

Shi-Yuan $\mathrm{Li}^{1,2}$, Qiu-Xiang Cheng ${ }^{3}$, Jia-Kun Liu ${ }^{1,2}$, Xiao-Qun Nie ${ }^{1,2}$ Guo-Ping Zhao ${ }^{1,4}$ and Jin Wang (D)

${ }^{1}$ Key Laboratory of Synthetic Biology, Institute of Plant Physiology and Ecology, Shanghai Institutes for Biological Sciences, Chinese Academy of Sciences, 200032 Shanghai, China; ${ }^{2}$ University of Chinese Academy of Sciences, 100049 Beijing, China; ${ }^{3}$ Shanghai Tolo Biotechnology Company Limited, 200233 Shanghai, China and ${ }^{4}$ Department of Microbiology and Li KaShing Institute of Health
Sciences, The Chinese University of Hong Kong, Prince of Wales Hospital, Shatin, New Territories, Shatin, New Territories, China Correspondence: Qiu-Xiang Cheng (qxcheng@tolobio.com) or Jin Wang (wangj01@hotmail.com)

\section{REFERENCES}

1. Zetsche, B. et al. Cpf1 is a single RNA-guided endonuclease of a class 2 CRISPRCas system. Cell 163, 759-771 (2015).

2. Li, S. Y., Zhao, G. P. \& Wang, J. C-brick: a new standard for assembly of biological parts using Cpf1. ACS Synth. Biol. 5, 1383-1388 (2016).

3. Lei, C. et al. The CCTL (Cpf1-assisted Cutting and Taq DNA ligase-assisted Ligation) method for efficient editing of large DNA constructs in vitro. Nucleic Acids Res. 45, e74 (2017).

4. Yang, H., Gao, P., Rajashankar, K. R. \& Patel, D. J. PAM-dependent target DNA recognition and cleavage by C2c1 CRISPR-Cas endonuclease. Cell 167, 1814-1828 (2016).

5. Swarts, D. C., van der Oost, J. \& Jinek, M. Structural basis for guide RNA processing and seed-dependent DNA targeting by CRISPR-Cas12a. Mol. Cell 66, 221-233 (2017).

6. Stella, S., Alcon, P. \& Montoya, G. Structure of the Cpf1 endonuclease R-loop complex after target DNA cleavage. Nature 546, 559-563 (2017).

7. Fonfara, I., Richter, H., Bratovic, M., Le Rhun, A. \& Charpentier, E. The CRISPRassociated DNA-cleaving enzyme $\mathrm{Cpf} 1$ also processes precursor CRISPR RNA. Nature 532, 517-521 (2016).

8. Yamano, T. et al. Crystal structure of Cpf1 in complex with guide RNA and target DNA. Cell 165, 949-962 (2016).

9. Dong, D. et al. The crystal structure of $\mathrm{Cpf} 1$ in complex with CRISPR RNA. Nature 532, 522-526 (2016)

10. Liu, L. et al. Two distant catalytic sites are responsible for $\mathrm{C} 2 \mathrm{C} 2$ RNase activities. Cell 168, 121-134 (2017).

11. East-Seletsky, A. et al. Two distinct RNase activities of CRISPR-C2c2 enable guideRNA processing and RNA detection. Nature 538, 270-273 (2016).

12. Abudayyeh, O. O. et al. $\mathrm{C} 2 \mathrm{C} 2$ is a single-component programmable RNA-guided RNA-targeting CRISPR effector. Science 353, aaf5573 (2016)

13. Gootenberg, J. S. et al. Nucleic acid detection with CRISPR-Cas $13 \mathrm{a} / \mathrm{C} 2 \mathrm{c} 2$. Science 356, 438-442 (2017).

\footnotetext{
Open Access This article is licensed under a Creative Commons cc) Attribution 4.0 International License, which permits use, sharing, appropriate credit to the original author(s) and the source, provide a link to the Creative Commons license, and indicate if changes were made. The images or other third party material in this article are included in the article's Creative Commons license, unless indicated otherwise in a credit line to the material. If material is not included in the article's Creative Commons license and your intended use is not permitted by statutory regulation or exceeds the permitted use, you will need to obtain permission directly from the copyright holder. To view a copy of this license, visit http://creativecommons. org/licenses/by/4.0/.
}

(c) The Author(s) 2018 